\title{
Prosthetic Joint Infection Following Total Hip Arthroplasty-Effect of Choice of Bearing Surfaces and How to Investigate Further-A Review of the Literature
}

\author{
Ahmed Saad, Shafiq A. Shahban \\ Heartlands Hospital, Birmingham, UK \\ Email: shafiqshahban@hotmail.com
}

How to cite this paper: Saad, A. and Shahban, S.A. (2018) Prosthetic Joint Infection Following Total Hip Arthroplasty-Effect of Choice of Bearing Surfaces and How to Investigate Further-A Review of the Literature. Open Access Library Journal, 5: e4663. https://doi.org/10.4236/oalib.1104663

Received: May 17, 2018

Accepted: June 17, 2018

Published: June 20, 2018

Copyright $\odot 2018$ by authors and Open Access Library Inc.

This work is licensed under the Creative Commons Attribution International License (CC BY 4.0).

http://creativecommons.org/licenses/by/4.0/

\begin{abstract}
Introduction: Total Hip Arthroplasty (THA) has proved to improve a patient's quality of life. This procedure has become more refined overtime, and subsequently there is huge variation in its execution. Prosthetic Joint Infection (PJI) remains a disastrous complication following this procedure. Main body: This article reviews the literature surrounding the various bearing surface options and fixation methods associated with the development of PJI. The current literature is reviewed to best describe how to investigate PJI following THA. Discussion: There is evidence supporting that no particular combination of articulating surfaces increases the risk of developing PJI. Cement, impregnated with antibiotics, has been proven to have a lower PJI rate over uncemented prostheses. Conclusion: Further research is required to best determine the optimum choice of bearing surfaces and fixation methods. If PJI is diagnosed, the cornerstone to its management is through a Multi-Disciplinary Team (MDT) approach.
\end{abstract}

\section{Subject Areas}

Radiology \& Medical Imaging

\section{Keywords}

Prosthetic Joint Infection, Infection, Total Hip Replacement, Arthroplasty, Bearing Surfaces

\section{Introduction}

Total hip arthroplasty (THA) is an operation that has continued to evolve over- 
time. When talking about the evolution of THA, one cannot forget to mention Sir John Charnley, who in the 1960's introduced low friction arthroplasty and whose research has led to the developments and novel techniques that we see today. Substantial amounts of research have been conducted to improve failure rates following THA, and even more so with the prevention of post-operative Prosthetic Joint Infection (PJI).

PJI is a serious complication following THA, which can have a significant impact(s) on patient morbidity, and, in some circumstances, can be fatal. With the best of efforts, the rate of developing PJI is between $1 \%-2 \%$ of all cases [1]. Although the literature reports multiple obscure organisms which can cause PJI, the usual suspects for this complication continue to be Staphylococcus aureus and Coagulase Negative Staphylococcus, which have been shown to account for up to $50 \%-75 \%$ of all cases [2].

Although many risk factors have been identified as contributing factors towards PJI, there is growing evidence which supports the theory that the choice of bearing surfaces and/or fixation method may influence this risk. When referring to the different bearing surfaces, the common options remain either metal-on-metal $(\mathrm{MoM})$, metal-on-polyethylene (MoP), ceramic-on-polyethylene $(\mathrm{CoP})$, or ceramic-on-ceramic ( $\mathrm{CoC})$. When choosing the fixation method and implanting one can choose to cement both acetabular and femoral components, without cement (uncemented), or to consider a hybrid approach. As of yet, there is no clear consensus to the ideal bearing materials and fixation methods.

\section{Main Body}

\section{Bearing surfaces}

One must not underestimate the importance of choosing bearing surfaces for THA. The choice from the various combinations available is an important one and one that can have significant lasting effects on the patient.

In a report by Madanat et al., they analysed 177,237 cases of THA taken from the Australian Registry (AOAN-JRR), and concluded that, between 1999 and 2013, (CoC) bearings had a lower revision rate for infection when compared to both $\mathrm{MoP}$ and $\mathrm{CoP}$. They also found no significant difference in infection rates between $\mathrm{CoP}$ and $\mathrm{MoP}$ articulating surfaces [3].

A similar study was undertaken in the USA between 2005 and 2014 and included a total of 315,784 elderly patients. This study, did not show a significant difference between $\mathrm{CoC}$ and $\mathrm{CoP}$ for PJI, but instead concluded that for primary THA with CoP and CoC bearings, there was significantly reduced risk of infection relative to MoP surfaces [4].

$\mathrm{Hu}$ et al. meta-analysed the literature and compared $\mathrm{CoC}$ and CoP only, focusing on the short-term to mid-term follow up. His findings concluded no significant difference between them with respect to loosening, revision and deep infection, following clinical and radiographic follow up [5]. Pitto et al. conducted a prospective study in New Zealand and demonstrated that the rate of 
early PJI ( $<6$ months) did not appreciably differ by bearing surface; however, in the long-term, $\mathrm{CoC}$ hips were associated with a lower risk of revision for deep infection when compared with CoP, MoP and MoM surfaces [6].

Despite the above, Hexter et al. conducted a systematic review and meta-analysis of 2272 studies and showed no significant difference between the combinations of articulating surfaces and the development of PJI [7].

\section{Fixation methods}

The choice between cemented vs. uncemented procedures has long been disputed by orthopaedic surgeons for both hemi and total hip arthroplasty. Cement has the added benefit of being able to be impregnated with antibiotics, with the aim of reducing the risk of PJI. However, bone cement implantation syndrome (BCIS) is only one of the recognised complications of using cement.

Engesæter et al. analysed the Norwegian registry of 56,275 cemented and uncemented primary THAs, and showed a slightly reduced rate of infection when antibiotic impregnated cement was used [8]. Yoon et al. demonstrated an increase in rates of infection following cemented THA, however, no mention was made with regards to if the cement used was impregnated with antibiotics or not [9].

Bone cement during the preparation and implantation phases, reacts exothermically. This heat can lead to osteonecrosis, and ultimately lead to a breeding ground for organisms, a hypothesis put forward by Minakawa et al. [10].

\section{PJI- how and when to appropriately investigate}

The first stages in the management of suspected PJI requires a thorough history and examination of the patient. Further investigations, both non-invasive and invasive, can be executed accordingly depending on how likely the risk of PJI is deemed at every stage. At the onset of this suspected diagnosis, the patient should be managed with a Multi-Disciplinary Team (MDT) approach.

Haematological investigations are relatively easy to perform, have a quick turnaround time and are generally well accepted from the patient. Inflammatory markers can be of use when investigating for PJI, however a normal result(s) can be misleading. A raised Erythrocyte Sedimentation Rate (ESR) or C-Reactive Protein (CRP) is suggestive of PJI [11] [12].

When it comes to imaging, plain radiographs are frequently unhelpful when trying to exclude PJI [13]. Other studies such as Ultrasound, CT and MRI scans can be helpful when looking for specific diagnoses. Equally a bone scan may be of use when trying to look at cellular activity in relation to infection/oedema. One study by Love et al. has found that leukocyte/marrow imaging is especially useful when looking for a hip PJI. The theory behind this was that white cells (associated with infection/inflammation) would tend to accumulate at sites of infection [14].

Other studies have looked at 18F-fluoro-deoxyglucose Positron Emission Tomography (PET) and have shown promising results, however, more research needs to be done before this could become a routine mode of investigation [12]. 
Further to this, aspiration of the joint in question would be the next step in the investigation of PJI. Further analysis by mean of a White Blood Cell (WBC) count and a percentage of Polymorphonuclear (PMN) cells has shown to have $84 \%$ and $82 \%$ sensitivity, respectively [15]. If undertaking this procedure, the use of a blood culture bottle can help in the detection of slow growing organisms, and the time between obtaining the sample and sending it to the lab should not be delayed, as this can influence the results accuracy [16].

\section{Discussion}

When selecting bearing surfaces for THA, the jury is still out with regards to which combination will help to reduce the risk of PJI. PJI aside, their other factors to consider, namely wear properties, patient functional demand, metal allergy, implant availability, financial implications, and, what we are seeing more commonly, patient preference - points of importance and beyond the scope of this article.

The practice of using cement in THA is debated amongst surgeons. Our findings demonstrate that when trying to limit the risk of PJI, then one should consider using antibiotic impregnated cement, or not at all.

Early appropriate investigation is key in helping to diagnose PJI, and to give the patient the best chance of a full recovery. The key in trying to get to this diagnosis starts simply with a thorough history and examination, and early involvement of the MDT.

\section{Conclusions}

Several studies have looked into bearing surfaces as well as fixation methods to try and investigate their association with the development of PJI. The jury still remains out with regards to the bearing surfaces and fixation methods which reduce the risk of PJI. Consideration of PJI is important, as are the biomechanical properties attached to the different bearing surfaces availablely.

Whatever the bearing surfaces and/or fixation methods, PJI can and does still prove to be challenge for the responsible clinician. A thorough workup and MDT approach are the gold standard when it comes to managing such a patient.

\section{References}

[1] Zimmerli, W., Trampuz, A. and Ochsner, P. (2004) Prosthetic-Joint Infections. New England Journal of Medicine, 351, 1645-1654.

https://doi.org/10.1056/NEJMra040181

[2] Sharma, D., Douglas, J., Coulter, C., Weinrauch, P. and Crawford, R. (2008) Microbiology of Infected Arthroplasty: Implications for Empiric Peri-Operative Antibiotics. Journal of Orthopaedic Surgery, 16, 339-342. https://doi.org/10.1177/230949900801600314

[3] CeraNews (2016) Plochingen: CeramTec GmbH Medical Products Division. 1st Edition, Springer Medizin, Berlin, 10-13.

http://poitr.pl/files/CeraNews_1-2016_US.pdf 
[4] Kurtz, S., Lau, E., Baykal, D. and Springer, B. (2017) Outcomes of Ceramic Bearings After Primary Total Hip Arthroplasty in the Medicare Population. The Journal of Arthroplasty, 32, 743-749. https://doi.org/10.1016/j.arth.2016.08.038

[5] Hu, D., Yang, X., Tan, Y., Alaidaros, M. and Chen, L. (2015) Ceramic-on-Ceramic versus Ceramic-on-Polyethylene Bearing Surfaces in Total Hip Arthroplasty. Orthopedics, 38, e331-e338. https://doi.org/10.3928/01477447-20150402-63

[6] Pitto, R. and Sedel, L. (2016) Periprosthetic Joint Infection in Hip Arthroplasty: Is There an Association between Infection and Bearing Surface Type? Clinical Orthopaedics and Related Research ${ }^{\circledR}, 474,2213-2218$. https://doi.org/10.1007/s11999-016-4916-y

[7] Hexter, A., Hislop, S., Blunn, G. and Liddle, A. (2018) The Effect of Bearing Surface on Risk of Periprosthetic Joint Infection in Total Hip Arthroplasty. The Bone \& Joint Journal, 100-B, 134-142.

https://doi.org/10.1302/0301-620X.100B2.BJJ-2017-0575.R1

[8] Engesæter, L., Espehaug, B., Lie, S., Furnes, O. and Havelin, L. (2006) Does Cement Increase the Risk of Infection in Primary Total Hip Arthroplasty? Revision Rates in 56,275 Cemented and Uncemented Primary THAs Followed for 0 - 16 Years in the Norwegian Arthroplasty Register. Acta Orthopaedica, 77, 351-358. https://doi.org/10.1080/17453670610046253

[9] Yoon, B., Ha, Y., Lee, Y. and Koo, K. (2015) Postoperative Deep Infection after Cemented versus Cementless Total Hip Arthroplasty: A Meta-Analysis. The Journal of Arthroplasty, 30, 1823-1827. https://doi.org/10.1016/j.arth.2015.04.041

[10] Minakawa, H., Stone, M., Wroblewski, B., Lancaster, J., Ingham, E. and Fisher, J. (1998) Quantification of Third-Body Damage and Its Effect on UHMWPE Wear with Different Types of Femoral Head. The Journal of Bone and Joint Surgery, 80, 894-899. https://doi.org/10.1302/0301-620X.80B5.8675

[11] Ghanem, E., Antoci, V., Pulido, L., Joshi, A., Hozack, W. and Parvizi, J. (2009) The Use of Receiver Operating Characteristics Analysis in Determining Erythrocyte Sedimentation Rate and C-Reactive Protein Levels in Diagnosing Periprosthetic Infection Prior to Revision Total Hip Arthroplasty. International Journal of Infectious Diseases, 13, e444-e449. https://doi.org/10.1016/j.ijid.2009.02.017

[12] Chryssikos, T., Parvizi, J., Ghanem, E., Newberg, A., Zhuang, H. and Alavi, A. (2008) FDG-PET Imaging Can Diagnose Periprosthetic Infection of the Hip. Clinical Orthopaedics and Related Research, 466, 1338-1342.

https://doi.org/10.1007/s11999-008-0237-0

[13] Peel, T., Buising, K. and Choong, P. (2010) Prosthetic Joint Infection: Challenges of Diagnosis and Treatment. ANZ Journal of Surgery, 81, 32-39. https://doi.org/10.1111/j.1445-2197.2010.05541.x

[14] Love, C., Marwin, S. and Palestro, C. (2009) Nuclear Medicine and the Infected Joint Replacement. Seminars in Nuclear Medicine, 39, 66-78. https://doi.org/10.1053/j.semnuclmed.2008.08.007

[15] Schinsky, M., Della Valle, C., Sporer, S. and Paprosky, W. (2008) Perioperative Testing for Joint Infection in Patients Undergoing Revision Total Hip Arthroplasty. The Journal of Bone and Joint Surgery-American Volume, 90, 1869-1875. https://doi.org/10.2106/JBJS.G.01255

[16] Yee, D., Chiu, K., Yan, C. and Ng, F. (2013) Review Article: Joint Aspiration for Diagnosis of Periprosthetic Infection. Journal of Orthopaedic Surgery, 21, 236-240. https://doi.org/10.1177/230949901302100225 